\title{
Des images et des douleurs
}

\section{Images and pains}

\section{Garcia-Larrea • R. Peyron}

C) Springer-Verlag France 2014

Au début du $\mathrm{xx}^{\mathrm{e}}$ siècle, Head and Holmes ont observé que les lésions pariétales produisaient peu d'altérations de la sensation douloureuse, et sur cette base, ont considéré que le rôle du cortex cérébral dans l'expérience de la douleur était très limité [2]. Malgré des rapports contradictoires importants mais passés largement inaperçus [1,3], cette vision des choses a prévalu durant la majorité du siècle dernier, et ne fut abandonnée que vers les années 1990, surtout grâce à l'imagerie fonctionnelle du cerveau. La question a été dès lors celle des fonctions que l'on peut assigner aux différentes aires cérébrales activées en concomitance avec l'expérience nommée « douleur ». Dans ce volume de Douleur et Analgésie, nous avons souhaité vous proposer une vision transversale et actualisée de différents domaines dans lesquels l'imagerie fonctionnelle du cerveau humain contribue à la connaissance des mécanismes corticaux sous-tendant l'expérience douloureuse, et de ses altérations en cas de douleur clinique. Le lecteur trouvera des rapports sur les niveaux d'intégration corticale autorisant le passage de la nociception à la douleur consciente, et sur les dérèglements observés en cas de différents types de douleurs cliniques. Une introduction méthodologique aidera, nous l'espérons, à mieux appréhender la façon dont les chercheurs réussissent à donner un sens physiologique aux images, et un article abordant l'étude des récepteurs opioïdes servira à rappeler les capacités de l'imagerie fonctionnelle à approcher la neurobiologie « humide ». Enfin, le chapitre voué aux réponses cérébrales évoquées par la vision de la douleur d'autrui aidera à comprendre que la douleur humaine peut, et doit, être aussi interpersonnelle.

\section{Références}

1. Biemond A (1956) The conduction of pain above the level of the thalamus opticus. Arch Neurol Psychiatry 75:231-44

2. Head H, Holmes G (1911) Sensory disturbances from cerebral lesions. Brain 34:102-254

3. Marshall J (1951) Sensory disturbances in cortical wounds with especial reference to pain. J Neurol Neurosurg Psychiatr 14:187204

L. Garcia-Larrea

Head, central integration of pain, centre for neuroscience of Lyon, Inserm U1028, Chairman, European chapter clinical neurophysiology societies (EC-IFCN), hôpital neurologique, 59 boulevard Pinel, F-69003 Lyon, France

R. Peyron

Centre stéphanois de la douleur et service de neurologie, CHU de

Saint-Étienne et NeuroPain, CRNL, INSERM U1028, France 